\title{
Hydrophobic modification of bacterial cellulose using oxygen plasma treatment and chemical vapor deposition
}

\author{
Salomé Leal · Cecília Cristelo (1) - Sara Silvestre - Elvira Fortunato • \\ Aureliana Sousa - Anabela Alves • D. M. Correia - S. Lanceros-Mendez • \\ Miguel Gama
}

Received: 20 July 2019/Accepted: 16 January 2020/Published online: 24 January 2020

(C) Springer Nature B.V. 2020

\begin{abstract}
A new strategy for the surface modification of bacterial cellulose (BC) through the combination of oxygen plasma deposition and silanization with trichloromethyl silane (TCMS) is described. The combined use of the two techniques modifies both the surface roughness and energy and therefore maximizes the obtained hydrophobic effect. These modified membranes were characterized by Scanning Electron Microscopy (SEM), water contact angle measurements, Fourier-transform infrared spectroscopy (FTIR-ATR)
\end{abstract}

S. Leal · C. Cristelo $(\bowtie) \cdot$ A. Alves · M. Gama Centre of Biological Engineering, University of Minho, Braga, Portugal

e-mail: ceciliacristelo3@gmail.com

S. Silvestre $\cdot$ E. Fortunato

Universidade Nova de Lisboa, Faculdade de Ciências e

Tecnologias, Lisbon, Portugal

A. Sousa

INEB - Biomedical Engineering Institute, Porto, Portugal

D. M. Correia $\cdot$ S. Lanceros-Mendez

Centro/Departamento de Física, University of Minho,

4710-057 Braga, Portugal

S. Lanceros-Mendez

BCMaterials, Basque Center for Materials, Applications

and Nanostructures, UPV/EHU Science Park,

48940 Leioa, Spain

S. Lanceros-Mendez

IKERBASQUE, Basque Foundation for Science,

48013 Bilbao, Spain and X-ray photoelectron spectroscopy (XPS), and its cytotoxic potential was investigated using both indirect and direct contact in vitro studies. The obtained results suggest an effective conjugation of TCMS to the surface of BC, leading to a highly hydrophobic surface, with a water contact angle of approximately $130^{\circ}$. It is also demonstrated that this is a stable and durable surface modification strategy, since $\mathrm{BC}$ remained hydrophobic even after 6 months, in dry conditions or after being submerged in distilled water for about a month. Importantly, this surface modification revealed no short-term cytotoxic effects on L929 and hDNFs cells. Altogether, these data indicate the successful development of a surface modification method that can be applied to $\mathrm{BC}$, enabling the production of a biodegradable and hydrophobic platform that can be applied to different areas of research and industry. 


\section{Graphic abstract}
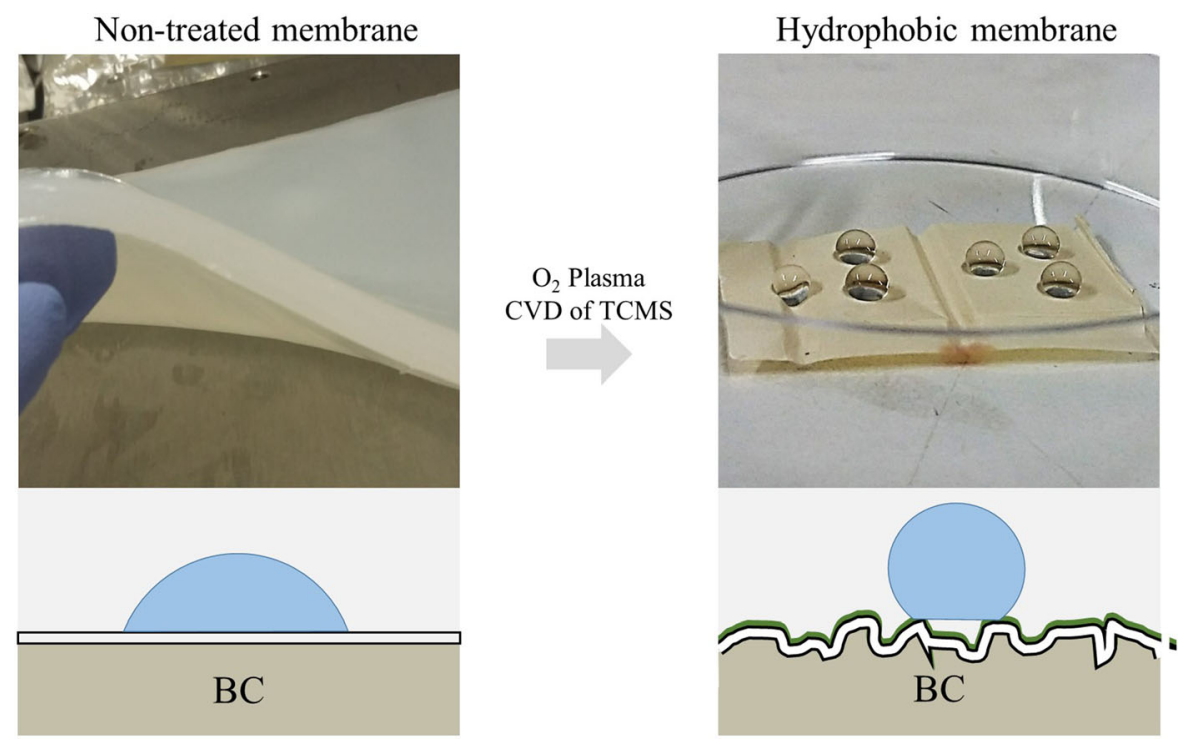

Keywords Bacterial cellulose $\cdot$ Chemical vapor deposition - Trichloromethylsilane - Oxygen plasma . Hydrophobic $\cdot$ Surface modification

\section{Introduction}

Cellulose is a highly abundant biopolymer, which is present mainly in plants (Klemm et al. 2005). Cellulose obtained from some bacteria, bacterial cellulose (BC), consists of a translucent and gelatinous film, produced by oxidative fermentation. Bacteria from the genera Komagataeibacter are the most efficient cellulose producers (Jedrzejczak-Krzepkowska et al. 2016). BC consists of a ultrafine network of wellarranged long cellulose microfibrils composed of $(1 \rightarrow 4)$-D-anhydroglucopyranose chains bounded through $\beta$-glyosidic linkages and reinforced by an inter and intramolecular hydrogen-bonding network (Picheth et al. 2017). BC presents many advantages with regards to the vegetal counterpart, given its purity (Petersen and Gatenholm 2011; Picheth et al. 2017), mechanical strength (Shao et al. 2017), elasticity, and biocompatibility (Costa et al. 2017).

Depending on the fermentation process and conditions used, the shape of $\mathrm{BC}$ can be manipulated to obtain different forms, size, thickness and crystallinity, making $\mathrm{BC}$ adaptable to diverse applications (Picheth et al. 2017).

Taking advantage of $\mathrm{BC}$ natural properties, $\mathrm{BC}$ has been explored in the biomedical field, as a wound dressing material (Huang et al. 2014), as an anticoagulant agent (Fink et al. 2010) and as a scaffold for bone regeneration (Zimmermann et al. 2011), among other applications. In recent years, the relevance of $\mathrm{BC}$ increased in several fields of study as a flexible matrix to develop nanocomposites and materials with tailored properties for electronic or magnetic applications (Cacicedo et al. 2016). Although the use of BC has extended to different fields, the high hydrophilicity of this biopolymer (although desirable in many instances) can limit its range of applications. Therefore, a surface modification method that can modify the surface chemistry of $\mathrm{BC}$ would be beneficial to fully exploit the applicability of this outstanding biopolymer. Hydrophobization of $\mathrm{BC}$ sets grounds for several other applications including printing, food packaging and storage, microfluidic and bioassay devices (Song and Rojas 2013).

The wettability of a surface depends on its surface energy and roughness (Cassie and Baxter 1944; Wenzel 1936) and, therefore, different methods have been investigated to tailor materials hydrophobic characteristics. These strategies include the use of fluorocarbons, silicones and organic or inorganic materials, which by grafting, adsorption or chemical- 
vapor deposition (CVD) have been applied to cellulose and decrease surface energy (Song and Rojas 2013). To obtain a stronger hydrophobic effect, methods which increase surface roughness can also be used prior to the surface energy reduction treatment, and these strategies include the use of electrospinning or electrospraying, nanoparticle deposition and plasma treatment (Calvimontes et al. 2011; Song and Rojas 2013).

Due to the high content of hydroxyl groups at the surface of $\mathrm{BC}$, the reaction with fluorinated or alkylchlorosilylating agents can readily occur in the gas-solid interface, using CVD. Using this strategy, the surfaces are exposed to the chemical in its vapor phase and the hydrophobic molecules are grafted onto the BC surface (Fadeev and McCarthy 2000). This concept has been already employed for the modification of paper's surface chemistry (Glavan et al. 2013). Since BC presents a higher mechanical strength and purity relative to paper, surface modification of this biopolymer can enable the production of materials with a wide range of possible applications, including as a platform for the design of microfluidic devices (Costa et al. 2014). Therefore, the aim of this work is to establish a strategy to successfully modify BC surface, by combination of two methodologies, namely oxygen plasma deposition, which increases the roughness of $\mathrm{BC}$, and silanization using trichloromethyl silane (TCMS) to decrease the surface energy. Using this strategy, it is hypothesize to obtain a biocompatible and long-lasting hydrophobic biomaterial that can resist wettability and therefore, unveil a large range of applications.

\section{Materials and methods}

\section{Bacterial cellulose processing}

Bacterial cellulose membranes (HTK CO., LTD, Vietnam) with an initial thickness of $12-15 \mathrm{~mm}$ were purified by alkali treatment with $\mathrm{NaOH} 0.1 \mathrm{M}$ for 3 days (solution renewed every $12 \mathrm{~h}$ ), under gentle stirring and at room temperature (RT). Afterwards, membranes were exhaustively washed with distilled water until the washing solution reached the $\mathrm{pH}$ of distilled water. Some of the membranes were also subject to washing with $5 \%(\mathrm{w} / \mathrm{v})$ of sodium dodecyl sulphate (SDS; Sigma-Aldrich, St. Louis, MO, USA), for 5 days (solution renewed everyday), followed by extensive washing with distilled water, under agitation (100 rpm). This step was introduced only for the membranes used in the direct contact cell viability assay to further purify membranes by removing the remaining endotoxins and to avoid any impact on cell behavior (Leitao et al. 2016). After the washing steps, membranes were then sterilized by autoclaving at $121{ }^{\circ} \mathrm{C}, 1 \mathrm{bar}$, for $20 \mathrm{~min}$, prior to storage.

Bacterial cellulose solvent exchange and densification

The BC membranes were processed by solvent exchange with ethanol, in order to accelerate drying, since this process allows the mechanical integrity of the membrane to be better preserved and yields a smother surface (Thuo et al. 2014). Firstly, membranes were compressed between two aluminum plates for $30 \mathrm{~min}$, expelling the water entrapped in the BC network, until the thickness of the membrane was reduced by around $80 \%$; then the membranes were immersed in increasing concentrations of ethanol solutions $(25 \%, 50 \%, 75 \%$ and $100 \% \mathrm{v} / \mathrm{v})$, for around $2 \mathrm{~h}$ in each stage, and stored in absolute ethanol until further use. When needed, BC membranes were again compressed between aluminum plates for $30 \mathrm{~min}$ (final thickness of approximately $2.3-2.4 \mathrm{~mm}$ ). The densified membranes were allowed to dry at $37{ }^{\circ} \mathrm{C}$ for $24 \mathrm{~h}$. The obtained dried membranes are referred to as "Non-treated".

Oxygen plasma treatment of $\mathrm{BC}$ membranes

In this study, oxygen plasma treatment was performed to increase the roughness of the $\mathrm{BC}$ membranes (Calvimontes et al. 2011). $\mathrm{O}_{2}$ Plasma treatment was performed using a radio frequency $(40 \mathrm{MHz})$ plasma reactor (Zepto Diener Electronics). Samples were exposed to $\mathrm{O}_{2}$ plasma at a potency of $100 \mathrm{~W}$, for 15 min. During the treatment, the gas flow was adjusted in order to keep a constant pressure of $100 \mathrm{~Pa}$ inside the reactor.

Chemical vapour deposition of trichloromethylsilane (TCMS)

Silanization was conducted in a reduced pressure chamber at a temperature set at $95{ }^{\circ} \mathrm{C}$. The silanizing 
reagent, TCMS (86 $\mathrm{mM}$ solution in anhydrous toluene), was placed inside the chamber together with the plasma-treated $\mathrm{BC}$ membranes and set to react for $60 \mathrm{~min}$, at $-50 \mathrm{kPa}$. Following that period, the beaker containing the remaining TCMS (in toluene) was removed from the oven and replaced with only toluene which was left for $10 \mathrm{~min}$ for the washing of some of unbound TCMS upon toluene condensation on BC surface. After this, the membranes in the chamber, were subject to vacuum $(-50 \mathrm{kPa})$ for $5 \mathrm{~min}$ to further remove the toluene and obtain the dried BC membranes.

Fourier transform infrared: attenuated total reflectance (FTIR-ATR)

The chemical modifications were investigated by FTIR-ATR. FTIR-ATR spectra collected at room temperature over a scanning range of $600-4000 \mathrm{~cm}^{-1}$ with a resolution of $4.0 \mathrm{~cm}^{-1}$, using a JASCO FT/IR 4100-Fourier Transform Infrared Spectrometer.

X-ray photoelectron spectroscopy (XPS)

The XPS analysis was performed using a Kratos AXIS Ultra HSA with VISION software for data acquisition and CASAXPS software for analysis. For analysis, an achromatic $\mathrm{Al} \mathrm{K} \alpha$ X-ray source operating at $15 \mathrm{kV}$ $(90 \mathrm{~W})$ was used, and the spectrometer was operated in FAT mode with $40 \mathrm{eV}$ pass energy for ROI regions and $80 \mathrm{eV}$ for survey. Data acquisition was performed at a pressure below $1 \times 10^{-6} \mathrm{~Pa}$.

Scanning electron microscopy (SEM)

The surface morphology of the membranes was analysed using a FEG-SEM (Ultra-high resolution Field Emisson Gun Scanning Electron Microscopy), NOVA 200 Nano SEM (FEI Company), scanning electron microscope (SEM). All specimens were precoated with a conductive layer of $\mathrm{Au} / \mathrm{Pd}$.

\section{Profilometry}

The surface roughness was analyzed using profilometry. The profiler (Ambios XP-Plus 200 Stylus) with an analysis speed of $0.1 \mathrm{~mm} / \mathrm{s}$ and a stylus force of $1.0 \mathrm{mg}$ was used to scan $1 \mathrm{~mm}$ in the surface of each sample.
Wettability studies: static and dynamic water contact angles (CA)

To obtain the static and dynamic water CA and characterize the surface wettability, the DataPhysics OCA 15 Plus set up was used. The system is composed of a syringe placed vertically, coupled to an automated dispenser while a digital image acquisition system records the droplet's lateral profile. Throughout the study, $3 \mu \mathrm{L}$ of ultrapure water droplets were used as reference. The static CA analysis was performed with the embedded software (SCA 20) using the LaplaceYoung approximation model with at least three measurements per sample. The stability of the surface properties was investigated by measuring the static water CA, in properly stored samples, sealed in dry vessels at room temperature, for up to 6 months. For further evaluation of the stability and durability of the surface modification of $\mathrm{BC}$, the samples were also submerged in distilled water, for up to 28 days at room temperature. After drying, the static contact angles were measured once more.

The dynamic water CAs, namely the advancing and receding $\mathrm{CA}$, were measured by changing the volume of the droplet. In short, $3 \mu \mathrm{L}$ droplet was first placed on the surface and the volume of the droplet was gradually increased, while recording the advancing contact angle. The receding angle was measured in the same way, but in this case gradually decreasing the droplet volume. The difference between the advancing and receding contact angles gives information on the surface hysteresis. To demonstrate the high water adhesiveness of the BC-treated surface, photographs of the water droplet on BC surface were taken with a tilting angle of 0,90 and $180^{\circ}$ (DataPhysics OCA 15 Plus).

Membrane's processing for in vitro assays

In the mentioned cases, a washing step was performed, either after obtaining the NT or the PlasSil-treated BC, to decrease the cytotoxic potential. This was achieved by submerging the BC membranes in MilliQ water $\left(1 \mathrm{~mL} / \mathrm{cm}^{2}\right)$ for $24 \mathrm{~h}$ in agitation at $60 \mathrm{rpm}$, after which the membranes were allowed to dry overnight at room temperature. Washed and non-washed membranes were sterilized using ultraviolet (UV) light before the in vitro viability assays, $30 \mathrm{~min} / \mathrm{side}$. This procedure is described as acceptable for the removal of 
common pathogenic bacteria, being easy to perform and with no residual effects left on the surface (Katara et al. 2008).

\section{Cell culture conditions}

L929 immortalized mouse lung fibroblasts cell line was purchased from ATCC. L929 cells were routinely cultured in Eagle's Minimum Essential Medium (EMEM), supplemented with $10 \%$ horse serum (HS; both from ATCC) and 1\% penicillin:streptomycin (P/ $\mathrm{S}$; Merk, Darmstadt, Germany). Subcultures were performed by trypsinization and cells were used until passage 10. For the assays, L929 cells were seeded in 96-well plates, at a density of $0.5 \times 10^{5}$ cells $/ \mathrm{mL}$. To allow cell adhesion, the plates were incubated overnight in humidified atmosphere of $5 \% \mathrm{CO}_{2}$ at $37{ }^{\circ} \mathrm{C}$, before any treatment.

The hDNFs (human dermal neonatal fibroblasts; ZenBio) primary cells were routinely cultured in Dulbecco's Modified Eagle's Medium (DMEM) supplemented with $10 \%$ fetal bovine serum (FBS; Gibco), $1 \% \mathrm{P} / \mathrm{S}$ (Biowest) and 1\% amphotericin B (Capricorn Scientific) and kept at $37{ }^{\circ} \mathrm{C}$ in a humidified atmosphere of $5 \% \mathrm{CO}_{2}$, and used until passage 8 . For cytotoxicity assays, hDNFs were seeded in 24-well plates and incubated in $500 \mu \mathrm{L}$ of complete DMEM and allowed to reach approximately $80-90 \%$ confluence.

In vitro cytotoxicity evaluation: indirect and direct contact assays

The effect of the surface treatment of BC membranes on cell viability was assessed according to ISO 10993-5 (2009) standard, through indirect and direct contact viability assays.

For the indirect contact experiment, the cytotoxicity of leaching compounds from the membranes on the cellular metabolism was investigated using L929 cells. Extracts were obtained by incubating the sterilized membranes in sterile complete culture media $\left(3 \mathrm{~cm}^{2} / \mathrm{mL}\right)$ for $24 \mathrm{~h}$, at $37^{\circ} \mathrm{C}$ and agitation at $200 \mathrm{rpm}$. In addition to evaluating the effect from whole extracts $(100 \%)$ on cell viability, $50 \%$ extracts were also prepared by dilution in fresh culture media. Complete culture media subject to the same conditions, but with no membranes, was used as blank. Cell culture media with and without $30 \%$ DMSO were used as positive and negative control, respectively. Cell viability was evaluated by the 3-(4,5-dimethylthiazol2-yl)-2,5-diphenyltetrazolium bromide (MTT) assay after $24 \mathrm{~h}$ of incubation with extracts. Briefly, at the end of the experiment, culture media was removed and $50 \mu \mathrm{L}$ of $1 \mathrm{mg} / \mathrm{mL}$ MTT solution (in phenol Red free media) was added in each well and incubated for $2 \mathrm{~h}$ at $37{ }^{\circ} \mathrm{C}$, protected from light. In metabolically active cells, the tetrazolium compound is reduced forming a colored insoluble formazan product, which was solubilized with isopropanol $(100 \mu \mathrm{L} /$ well $)$, with color intensity being proportional to the number of viable cells (Mosmann 1983). Absorption was determined spectrophotometrically at $570 \mathrm{~nm}$ using a microplate reader spectrophotometer (Bioteck Plate Reader, Synergy MX) and cell viability expressed as percentage (\%) relative to a non-treated control.

For the direct contact viability assay, hDNFs primary cells were used. Samples (approximately $1 \mathrm{~cm}^{2}$ with less than $10 \%$ of weight variation) were directly put into the wells containing fibroblasts and the medium was refreshed, allowing the simultaneous test of the material itself and the leachable substances thereof. All experiments were carried out in triplicate and cells that were not in contact with the materials were used as control. The resazurin-based assay was used to assess cytotoxicity after $24 \mathrm{~h}$ incubation. Similar to the MTT reduction assay, the resazurinbased assay is able to monitor the cell's redox state, in which the active metabolism of viable cells can reduce resazurin into the pink and fluorescent product resorufin (Riss et al. 2016). Briefly, culture medium was removed and replaced with complete medium with $20 \%$ (v/v) resazurin (Sigma-Aldrich, Germany) and incubated at $37{ }^{\circ} \mathrm{C}$ in a humidified incubator with $5 \% \mathrm{CO}_{2}$ for $2 \mathrm{~h}$. Afterwards, $3 \times 100 \mu \mathrm{L}$ per well were transferred to a 96-well black plate/clear bottom and fluorescence was measured $(\lambda \mathrm{ex}=530 \mathrm{~nm} / \lambda \mathrm{em}=$ $590 \mathrm{~nm}$ ) using the aforementioned microplate reader spectrophotometer.

\section{Manufacturing of a BC-based platform}

A preliminary study was performed to address one of the possible applications of the hydrophobically modified BC. Hypothesizing the use of BC membranes as a platform for in vitro cell culture, $\mathrm{BC}$ was shaped upon drying, embossing the solvent-exchanged membrane between two complementary molds, which 
would imprint its shape on BC. Molds were designed using the Fusion 360 software and the 3D printer Ultimaker $2+$ to obtain the Polylactic Acid (PLA) thermoplastic moulds. These molds were intended to obtain the features of wells and channels which would enable the physical receptacle of cells, and the continuous feeding of cell culture media, and respective removal of excreted by-products. $\mathrm{BC}$ within the molds was dried at $37{ }^{\circ} \mathrm{C}$, for $40 \mathrm{~h}$ to complete the process and obtain the $3 \mathrm{D}$ membrane that was later subject to the surface modification treatments previously described.

\section{Statistical analysis}

The statistical analysis in this study was performed using the Graphpad Prism 6 software. For the cell viability studies, experiments were performed in triplicate, in three different independent assays and significant differences between the different treatments on cell viability were identified using unpaired $t$ test (2 tailed) for the indirect and direct contact viability assays.

\section{Results and discussion}

\section{Infrared vibration spectra}

In order to characterize the chemistry of the modified surface, Attenuated Total Reflection Fourier Transform infrared spectroscopy (FTIR-ATR) analysis was used. Figure 1 shows the FTIR-ATR spectra with the characteristic bands of native $\mathrm{BC}$, namely $\mathrm{O}-\mathrm{H}$ stretching at $3345 \mathrm{~cm}^{-1}, \mathrm{C}-\mathrm{H}$ stretching at $2900 \mathrm{~cm}^{-1}, \mathrm{CH}_{2}$ bending at $1420 \mathrm{~cm}^{-1}$, and $\mathrm{C}-\mathrm{O}-\mathrm{C}$ skeletal vibrations of pyranose ring at $1055 \mathrm{~cm}^{-1}$, as previously reported by Kumar et al. (2014). The peak assigned to $\mathrm{Si}-\mathrm{CH}_{3}$ at $1280 \mathrm{~cm}^{-1}$ (Mohd et al. 2016) indicates the presence of TCMS at the surface of BC. Bands for Si-O-Si and $-\mathrm{Si}-\mathrm{O}-\mathrm{C}$ bond at $1160 \mathrm{~cm}^{-1}$ and $1108 \mathrm{~cm}^{-1}$, respectively (Rachini et al. 2012), overlapped with band $\mathrm{C}-\mathrm{O}-\mathrm{C}$ corresponding to the cellulose skeletal vibration in the range $970-1250 \mathrm{~cm}^{-1}$, however these are slightly higher in the case of Sil and PlasSil samples.
Surface chemical modifications

XPS analysis was used to characterize the relative atomic concentration of carbon $(\mathrm{C})$, oxygen $(\mathrm{O})$ and silicon ( $\mathrm{Si}$ ) on the $\mathrm{BC}$ membranes obtained using the different surface modification treatments. The results from the XPS analysis are shown in Table 1. The nontreated and plasma-treated membranes reveal a similar $\mathrm{C}$ and $\mathrm{O}$ elemental profile. An increase in $\mathrm{Si}$ corresponds to the silanization treatment whether there was a pre-treatment with $\mathrm{O}_{2}$ plasma or not, the amount of $\mathrm{Si}(\%)$ being similar, which suggests that the efficiency in the conjugation of TCMS to BC does not depend on the $\mathrm{O}_{2}$ plasma pre-treatment. Therefore, since the deposition of TCMS appears to be similar in both treatments, the increased hydrophobicity obtained in the PlasSil treatment is explained by the increased roughness conferred by the $\mathrm{O}_{2}$ plasma pretreatment (Seo et al. 2015). As described by Wenzel (1936) the increased roughness contributes to higher hydrophilicity in a hydrophilic surface and to a higher hydrophobicity in a hydrophobic one. As expected, the increase in $\mathrm{Si}(\%)$ is accompanied by a decrease in the relative percentage of $\mathrm{C}$ and $\mathrm{O}$.

\section{Morphological features}

With the aim of increasing the BC surface hydrophobicity, samples were allowed to react with trichloromethyl silane (TCMS), after pre-treatment with $\mathrm{O}_{2}$ plasma. To characterize surface morphology of the produced BC membranes, samples were analysed by SEM. Figure 2 presents the scanning electron micrographs of the membranes at different stages of the process.

Scanning electron micrographs of the surface of non-treated and silanized samples without oxygen plasma pre-treatment revealed in general a smooth surface, with a well-defined and closed fibre network, without pores (Fig. 2a, c). The SEM analysis of the plasma-treated fibers, followed or not by silanization, revealed an increased roughness, open porosity and no discernible fiber network (Fig. 2b, d). This remarkable alteration in the BC membrane surface morphology is induced by the oxygen plasma treatment. Alteration in the roughness of cellulose, induced by oxygen plasma is, according to Calvimontes et al. (2011), caused by the decomposition of polymer chains and oxidation reactions. 


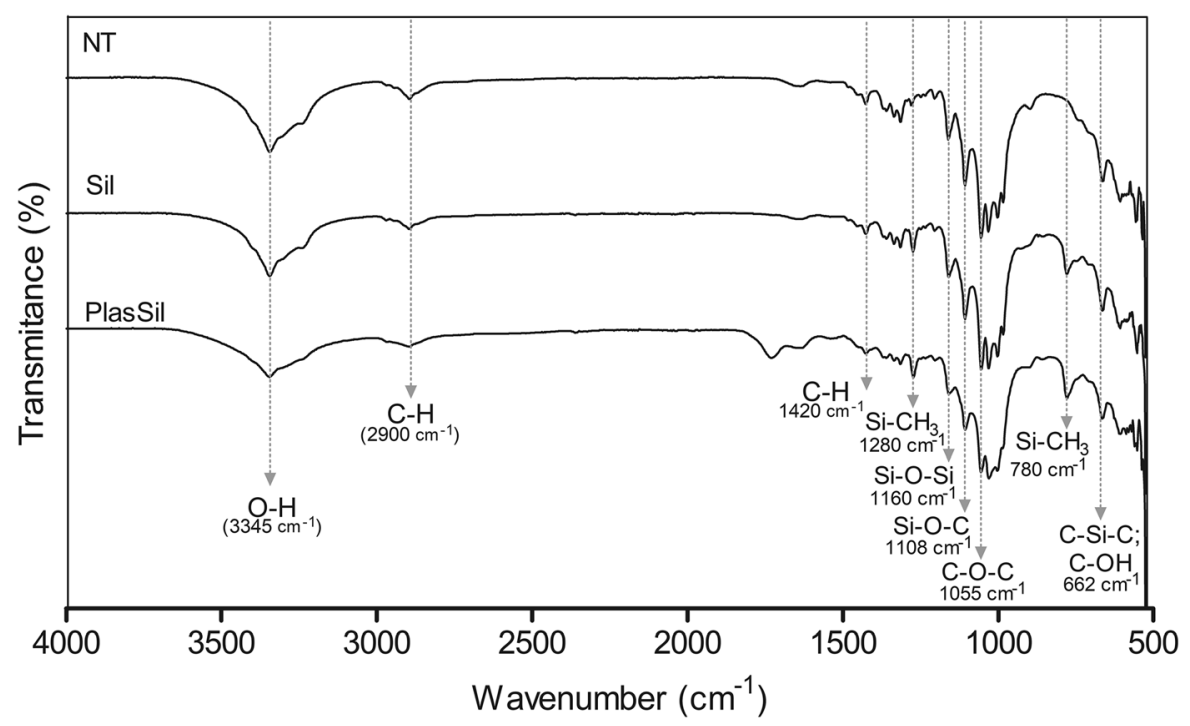

Fig. 1 FTIR spectra of BC membranes subject to different surface treatments (NT-Non-treated; Plas- $\mathrm{O}_{2}$ Plasma-treated; SilSilanized BC without plasma treatment; PlasSil—Silanized BC with $\mathrm{O}_{2}$ plasma pre-treatment

Table 1 BC membrane surface elemental composition obtained by XPS for Non-treated (NT) samples, $\mathrm{O}_{2}$ plasmatreated membranes (Plas), silanized membrane without plasma treatment (Sil) and silanized membrane with $\mathrm{O}_{2}$ plasma pretreatment (PlasSil)

\begin{tabular}{lllll}
\hline At $(\%)$ & & & & \\
\hline Element & NT & Plas & Sil & PlasSil \\
C 1s & 55.6 & 54.8 & 51.9 & 51.3 \\
N 1s & - & 0.8 & - & 0.5 \\
O 1s & 44.4 & 44.4 & 43.4 & 43.5 \\
Si 2p & - & - & 4.8 & 4.7 \\
\hline
\end{tabular}

The internal structure of $\mathrm{BC}$ was also analyzed using SEM, and the results shown in Fig. 3 demonstrate that the increased roughness induced by oxygen plasma treatment is confined to the surface and that the overall lamellar structure of the membranes is preserved.

\section{Surface profilometry}

To further characterize changes in the topographic roughness of $\mathrm{BC}$, samples were also analyzed using optical profilometry. With this analysis, the grooves and ridges of a surface are screened and the thickness, or distance of each point to the reference level, can be plotted. From the results shown in Fig. 4 it is noticeable that the non-treated and Sil-treated BC membranes present a similar and smoother roughness profile when compared with the PlasSil samples. PlasSil membranes showed a high variation of thickness throughout the analyzed area, which is indicative of a high roughness and higher surface area. These results are in agreement with the observed using SEM, and indicate that the $\mathrm{O}_{2}$ plasma treatment is essential for the alteration of surface roughness. When aiming at obtaining a hydrophobic surface, increasing surface roughness prior to the deposition of a hydrophobic coating leads to an increased hydrophobicity (Wenzel 1936). In this sense, it is expected that this treatment should improve the obtained hydrophobic effect.

Wettability studies

To analyze the effectiveness of the surface wettability modification method described in this work, the static and dynamic contact angles (CA) were measured. The static water $\mathrm{CAs}$ for the $\mathrm{BC}$ membranes with different surface modification are presented in Table 2.

The effect of the $\mathrm{O}_{2}$ plasma treatment is complex. On the one hand, it is likely to change the surface chemistry (nitrogen is detected in the plasma treated samples by XPS); on the other hand, the increased roughness associated to the low contact angle will generate capillary effects, since a concave meniscus 

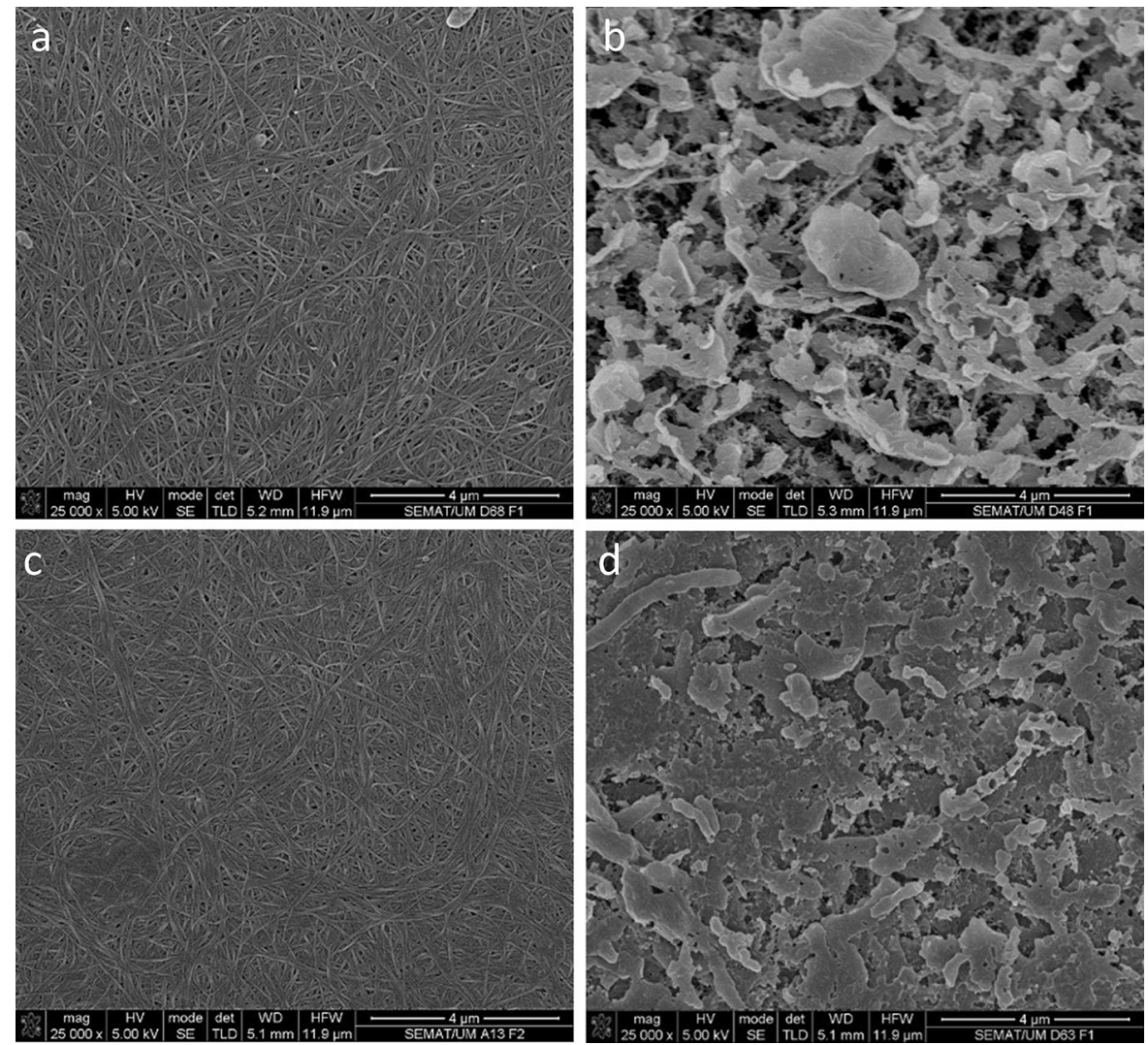

Fig. 2 SEM micrographs of the produced BC membranes. a Non-treated membrane, $\mathbf{b} \mathrm{O}_{2}$ plasma-treated membrane, $\mathbf{c}$ silanized membrane without $\mathrm{O}_{2}$ plasma treatment and d silanized membrane with $\mathrm{O}_{2}$ plasma pre-treatment $(\mathrm{mag} 25000 \times$, scale bar $=4 \mu \mathrm{m})$

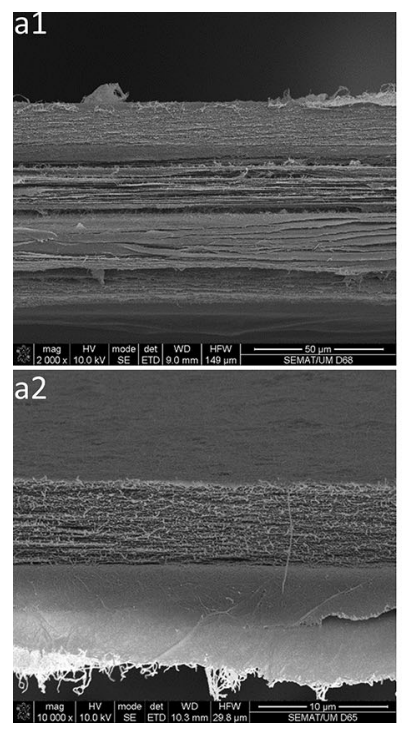

Fig. 3 SEM micrographs of the transversal section of the BC membranes. a Non-treated membrane, b $\mathrm{O}_{2}$ plasma-treated membrane, c silanized membrane without plasma treatment,
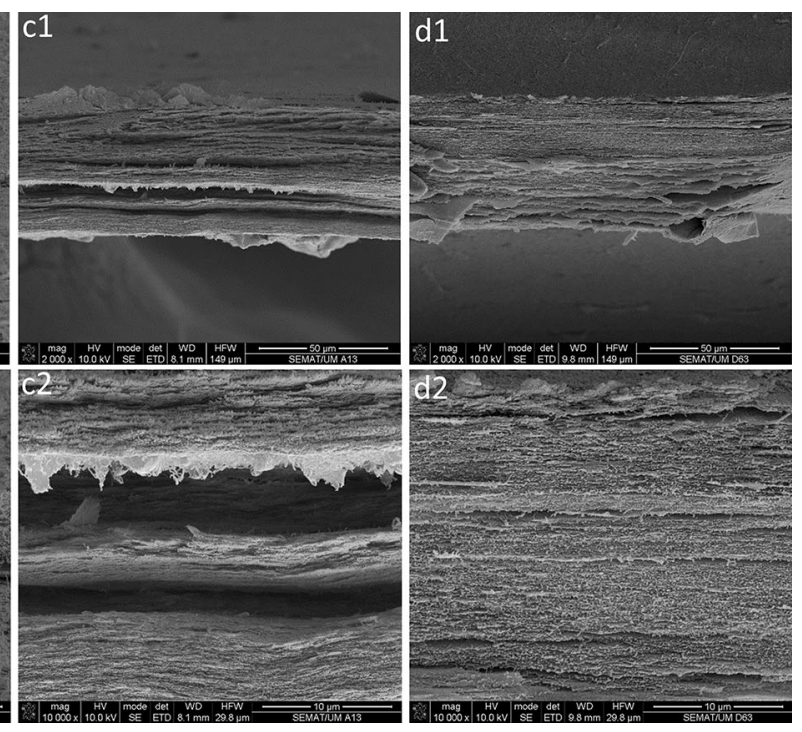

d silanized membrane with $\mathrm{O}_{2}$ plasma pre-treatment (1, scale bar $=50 \mu \mathrm{m} ; 2$, scale bar $=10 \mu \mathrm{m}$ ) 


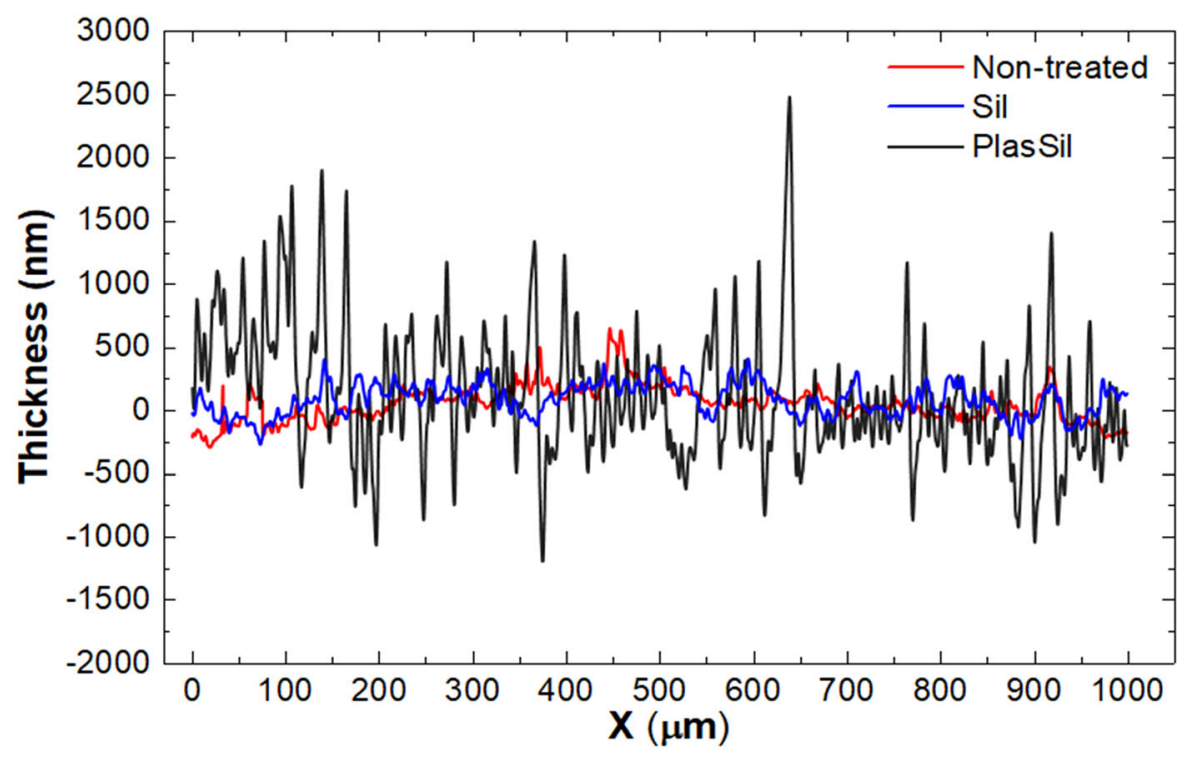

Fig. 4 Profilometry analysis of the non-treated BC membranes and the samples subject to silanization without plasma treatment (Sil) and silanization with $\mathrm{O}_{2}$ plasma pre-treatment

Table 2 Static water contact angles obtained for the different surface modification treatments of the BC membranes

\begin{tabular}{|c|c|c|}
\hline \multicolumn{2}{|l|}{ BC sample } & Initial CA \\
\hline \multicolumn{2}{|l|}{ NT } & $24.9 \pm 1.9$ \\
\hline \multicolumn{2}{|l|}{ Plas } & $26.6 \pm 1.2$ \\
\hline \multicolumn{2}{|l|}{ Sil } & $119.8 \pm 2.3$ \\
\hline \multicolumn{2}{|l|}{ PlasSil } & $132.6 \pm 7.2$ \\
\hline $\begin{array}{l}\text { Conditions } \\
\text { Stability as }\end{array}$ & \multicolumn{2}{|c|}{ Stability assay (PlasSil) } \\
\hline Dry & 180 & $135.4 \pm 1.2$ \\
\hline \multirow[t]{2}{*}{ Wet } & 7 & $110.3 \pm 14.5$ \\
\hline & 28 & $108.2 \pm 6.5$ \\
\hline
\end{tabular}

NT-Non-treated; Plas- $\mathrm{O}_{2}$ Plasma-treated; Sil—Silanized $\mathrm{BC}$ without plasma treatment; PlasSil-Silanized $\mathrm{BC}$ with $\mathrm{O}_{2}$ plasma pre-treatment

will arise, favoring wettability. However, the contact angle formed upon deposition of the water droplet $\left(26.6^{\circ}\right)$ does not significantly change, as compared with the non-treated material $\left(24.9^{\circ}\right)$. Differently, the treatment with TCMS on its own (Sil) is able to increase the static contact angle to $119.8^{\circ}$, due to the presence of the hydrophobic moieties of $\mathrm{Si}-\mathrm{CH}_{3}$, as detected by XPS and FTIR. Surface $\mathrm{O}_{2}$ plasma treatment previous to the TCMS silanization (PlasSil) further increases the contact angle to $132.6^{\circ}$. The higher hydrophobicity is explained by the formation of a convex meniscus, due to high contact angle, leading to air entrapment in the rough surface, which contributes to a higher contact angle and lower wettability of the surface, a phenomena well documented in the literature (Cassie and Baxter 1944).

Given the successful modification of BC wettability using the PlasSil treatment $\left(\mathrm{O}_{2}\right.$ plasma followed by silanization), the stability of the surface modification obtained after this method was investigated over time, under different conditions. Samples stored at room temperature were investigated by measuring the static CA after 6 months (180 days). The results shown in Table 2 demonstrate that the surface properties of the dry samples remain stable, with no significant changes in the CA over time.

The stability of the PlasSil surface modification was moreover investigated by submerging the membranes in distilled water up to 28 days at room temperature. This assay was essential to understand if the conjugation of TCMS to BC was effective and stable. The results reveal a significant decrease of the $\mathrm{CA}$ in the first 7 days of contact with distilled water, with a stabilization at a value around $110^{\circ}$ (Table 2). The decreased CA values together with the higher standard deviation suggests some leaching of the surface coating and a higher heterogeneity in the samples. 
Nevertheless the CA remained always high, the BC surface continuing highly hydrophobic (Song and Rojas 2013) even after 28 days submerged in water.

In rough surfaces, wettability should not be characterized just by static CA measurements. In fact, the droplets can present multiple equilibrium positions, and therefore there is a maximum $\mathrm{CA}$ value, known as advancing $\mathrm{CA}(\theta \mathrm{A})$ and a minimum $\mathrm{CA}$ value, which is called receding $C A(\theta R)$, with the difference between the two values being called CA hysteresis (Bhushan and Nosonovsky 2010). The results represented in Table 3 show that both surface modification strategies, Sil or PlasSil, result in a similar CA hysteresis around $85^{\circ}$. This high CA hysteresis is the result from $\mathrm{BC}$ surface roughness as well as from some chemical heterogeneity. Whereas the results shown in Fig. 2 show a remarkably different surface roughness after the Sil and PlasSil surface treatments, a similar CA hysteresis is obtained (Table 3).

The large difference between advancing and receding CAs may translate a high water adhesiveness, as can indeed be observed in this case, the water drop remaining adhered to $\mathrm{BC}$ surface even when the sample is rotated by 90 and $180^{\circ}$ (Fig. 5b, c). Water repellency associated with a low CA hysteresis means a very low roll-off angle, which is desirable in the engineering of self-cleaning microchannels for liquid flow applications, for example. This wetting regime is called the "Lotus effect" (Bhushan and Jung 2011). Although not intuitive, a hydrophobic surface can also display high water adhesion. When water penetrates into the micro-roughness, but not the nano-roughness, of a solid surface, another wetting regime exists which is known as the "petal effect", characterized by superhydrophobicity associated with a high water adhesion (Bhushan and Nosonovsky 2010). Superhydrophobic surfaces with high water adhesion have

Table 3 Contact Angle (CA) hysteresis obtained from the advancing $(\theta \mathrm{A})$ and receding water $\mathrm{CA}(\theta \mathrm{R})$ for the different surface modification treatments of $\mathrm{BC}$ : Sil-Silanized $\mathrm{BC}$ without plasma treatment; PlasSil-Silanized $\mathrm{BC}$ with $\mathrm{O}_{2}$ plasma pre-treatment

\begin{tabular}{llll}
\hline & $\theta \mathrm{A}$ & $\theta \mathrm{R}$ & CA hysteresis $\left(^{\circ}\right)$ \\
\hline Sil & $110 \pm 1$ & $26 \pm 3$ & 84 \\
PlasSil & $122 \pm 5$ & $36 \pm 4$ & 86 \\
\hline
\end{tabular}

potential for application in the transport of small liquid volumes, such as in open microfluidic devices (Hong et al. 2007). As previously mentioned, the advancing $\mathrm{CA}$ represents the maximum value for static $\mathrm{CA}$ in a heterogeneous and rough surface, however in the results obtained, a lower value for the advancing CA relative to de static $\mathrm{CA}$ is apparent which can be attributed to the sample's heterogeneity.

In vitro toxicity studies

To further investigate the applicability of this hydrophobically modified substrate, its effects on cell viability were investigated. An indirect contact assay was performed in which L929 cells were exposed to culture media containing the extracted leachable components from the PlasSil-modified or NT BC surface. The L929 cell line was used to screen the cytotoxicity of the developed membranes, since this is a well-established cell model (Faria et al. 2009). As depicted in Fig. 6, after $24 \mathrm{~h}$ in contact with the whole extracts $(100 \%)$ of unwashed NT membranes, there was a significant and unexpected reduction in cell viability. This cytotoxic effect was even more pronounced than in the unwashed PlasSil membranes. Given the purity of BC, and its demonstrated biocompatibility in vitro (Silva et al. 2018) as well as in vivo (Helenius et al. 2006), with good applicability as a scaffold for tissue engineering (Svensson et al. 2005), this cytotoxic effect of the NT membranes was initially attributed to insufficient washing of $\mathrm{BC}$ membranes. However, the deleterious effect from NT membranes on cell viability was later found to be associated with ethanol residues from the solventexchange stage. Sterilization using $70 \%$ (v/v) ethanol was exploited for the decontamination of the membranes used in the in vitro assays and, in all tested conditions, this resulted in a complete inhibition of cell viability, when compared to the effect from nonsterilized samples (results not shown). Therefore, this method was discontinued and UV radiation was used for membrane sterilization, with no visual signs of contamination throughout the assay.

Nonetheless, the solvent-exchange step was maintained, therefore in order to decrease the cytotoxic effects from ethanol and TCMS leachable components on cell viability, a washing step using MilliQ water was introduced before in vitro experiments in both NT and PlasSil-treated BC membranes. This resulted in a 
a

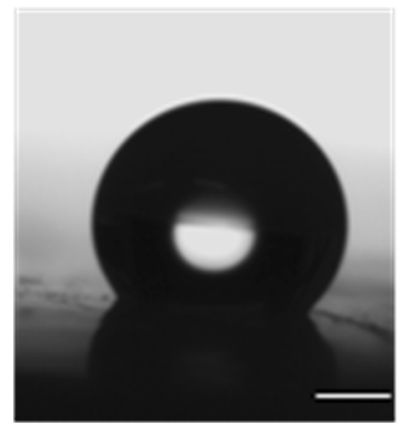

b

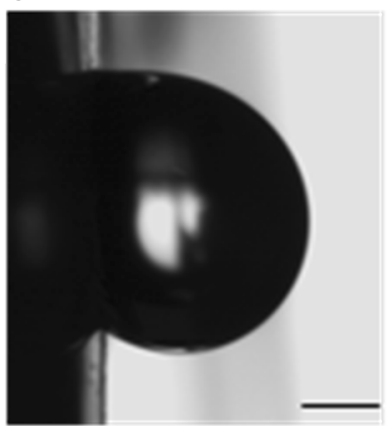

c

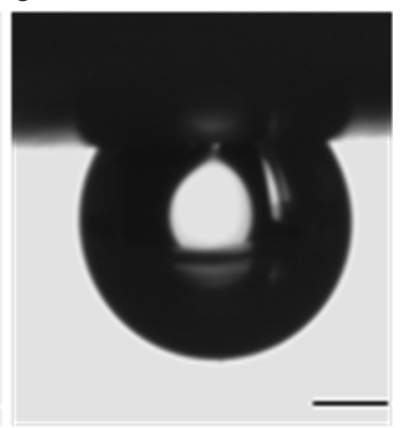

Fig. 5 Shape of droplets (MiliQ water) on the surface of PlasSil-modified BC at different tilting positions, at $0^{\circ}$ (a), $90^{\circ}(\mathbf{b})$ and $180^{\circ}$ (c) $($ Scale bar $=0.5 \mathrm{~mm})$

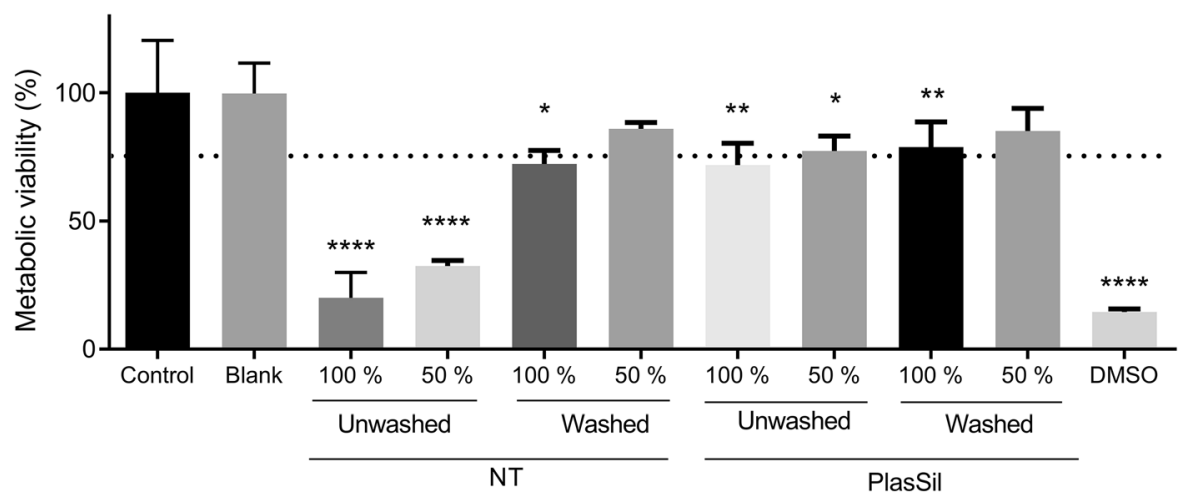

Fig. 6 Effects from the extracts of the hydrophobically modified BC on cell viability. L929 cells were incubated with the whole $(100 \%)$ or diluted $(50 \%)$ cell culture extracts, from either washed or unwashed non-treated (NT) or PlasSil-treated

significant improvement of cell viability in response to the diluted and whole extracts from NT membranes (Fig. 6). The fact that the unwashed PlasSil membranes, which could have leachable residues of TCMS and toluene, showed a lower cytotoxic effect than the unwashed NT membranes also corroborates the hypothesis that the presence of ethanol residues primarily accounts for the observed cytotoxic effects. Since the reaction with TCMS occurs at $95^{\circ} \mathrm{C}$, for $1 \mathrm{~h}$, this could allow a more efficient evaporation of ethanol residues. Therefore, washing after the PlasSil surface modification resulted a lower degree of improvement of cell viability. The whole extracts from washed PlasSil membranes caused a significant reduction on cell viability, noteworthy cell viability in these cases remained above $70 \%$, which can be considered as non-cytotoxic (ISO 10993-5 2009). As expected, the $50 \%$ extract had a lower effect on cell
BC membranes. Cell viability was evaluated using the MTT reduction assay after $24 \mathrm{~h}$. Cell viability is expressed in \% relative to the non-treated control $(* p \leq 0.05$; ** $p \leq 0.001)$

viability, with an innocuous effect, from the analysis performed. These results suggest that the developed surface treatment for the hydrophobization of BC has no short-term effects on cell viability.

A direct contact study was also performed to clarify any cytotoxic potential. This time, a primary cell culture was used, namely hDNFs. The use of a primary cell culture of fibroblasts, which is more sensitive than routinely used cell lines, phenotypically closer to its in vivo counterparts (Pan et al. 2009) and in this case, from human origin, can help to detect more accurately any cytotoxic effects from the PlasSil treatment. In this study, NT and PlasSil-modified BC membranes were directly brought into contact with cells and, as can be seen from Fig. 7, after $24 \mathrm{~h}$ with the washed and unwashed PlasSil-modified BC membranes, the cell viability remains very close to the control with no substantial alterations. It can also be noted that both 


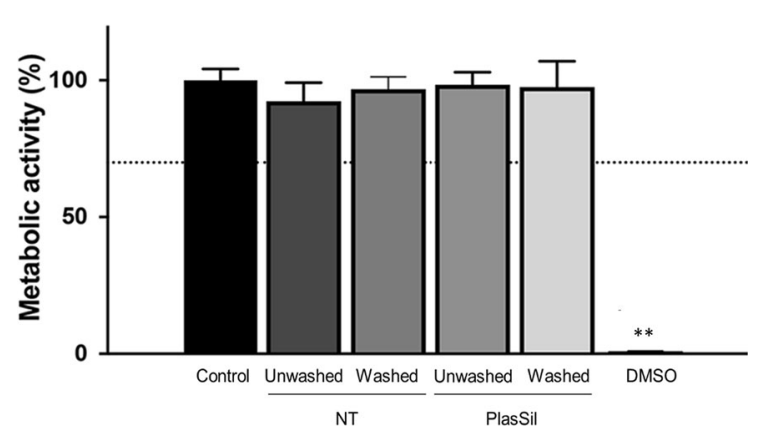

Fig. 7 Effects from the direct contact of hydrophobically modified $\mathrm{BC}$ membranes on cell viability. hDNFs primary cells were directly incubated with the non-treated (NT) or PlasSiltreated BC membranes. Cell viability was evaluated after $24 \mathrm{~h}$ by the resazurin-based assay and cell viability expressed in \% relative to the control $(* * p \leq 0.001)$

the unwashed and washed NT BC membranes showed no cytotoxic effect, contrary to what was observed in the indirect contact study. The extraction conditions of the indirect contact assay (high speed-200 rpm-and at $37^{\circ} \mathrm{C}$ ) attempt to simulate and exaggerate the leaching of the possible cytotoxic compounds present in a given material (ISO 10993-5 2009). Although the cell models used in the indirect and direct contact studies were different, in the indirect contact assay cells were exposed, from the beginning to the end of the experiment, to culture media containing the released compounds over a $24 \mathrm{~h}$ window, under the conditions needed to extract the majority, if not all, of the leaching compounds. On the other hand, in the direct contact study, cells are incubated in fresh media and the sample placed in the centre of the well containing the cells (ISO 10993-5 2009). This allows a simultaneous testing of direct contact toxicity and the leaching of compounds to media, however, leaching of any compounds is expected to be far more slow than it would be under agitation. Altogether, these results suggest that the direct contact of NT or PlasSil-treated $\mathrm{BC}$ membranes has an innocuous effect on cells, and that the leaching of compounds from BC can only be a concern in the case of intensified extraction conditions.

This is not the first report over the use of plasma treatment followed by silanization for cellulose surface modification. It was previously reported by Zhang et al. (2016) the use of Argon plasma and silanization by a physical-soaking method to obtain superhydrophobic surface of vegetable cellulose. Nevertheless this is, to our knowledge, the first report of the use of oxygen plasma followed by silanization using CVD. The use of CVD allows the easy, effective and reproducible surface modification, leaving impurities in solution out of the reaction and allowing the surface modification of shaped substrates (Creighton and Ho 2010).

Attempting to change the surface chemistry of paper, Glavan et al. (2013) proposed a simple and rapid method which consists in CVD of organosilanes, representing a technique able to modify vegetal cellulose to obtain surfaces with highly hydrophobic properties. In this case, we propose that the additional pre-treatment with oxygen plasma can enhance the hydrophobic effect obtained after silanization in BC, which is a more pure, resistant and biocompatible cellulose source. Given the reported effect of rough hydrophobic surfaces on improving cell adhesion (Wang et al. 2003), it is proposed that the PlasSil surface modification strategy is most suitable for the use of BC as a platform for cell culture.

The hydrophobic modification of cellulose described herein can also be subject to further modifications if needed. Namely, the concentration of TCMS can be increased, or the silanization duration can be prolonged to obtain higher CA measurements and a higher conjugation efficiency. Altogether, the results obtained regarding the exposure of cells to the PlasSil-treated BC membranes are very promising, showing a good biocompatibility in both direct and indirect contact experiments. Further studies are required, but the present one already shows the suitability of this biopolymer for applications as cell culture platform or in the design of microfluidic devices.

\section{Manufacturing of a BC-based platform}

Given the biocompatibility of the hydrophobic modification of BC herein shown and the current concern with plastic overuse (North and Halden 2013), the hypothesis that this biodegradable biomaterial could be used as a platform for the continuous cell culture was raised. Some preliminary studies were conducted in which the shape of $\mathrm{BC}$ was tailored to obtain the features of channels and wells, as represented in Fig. 8. This demonstrates the successful shaping of $\mathrm{BC}$ to obtain a platform which, due to the high water 


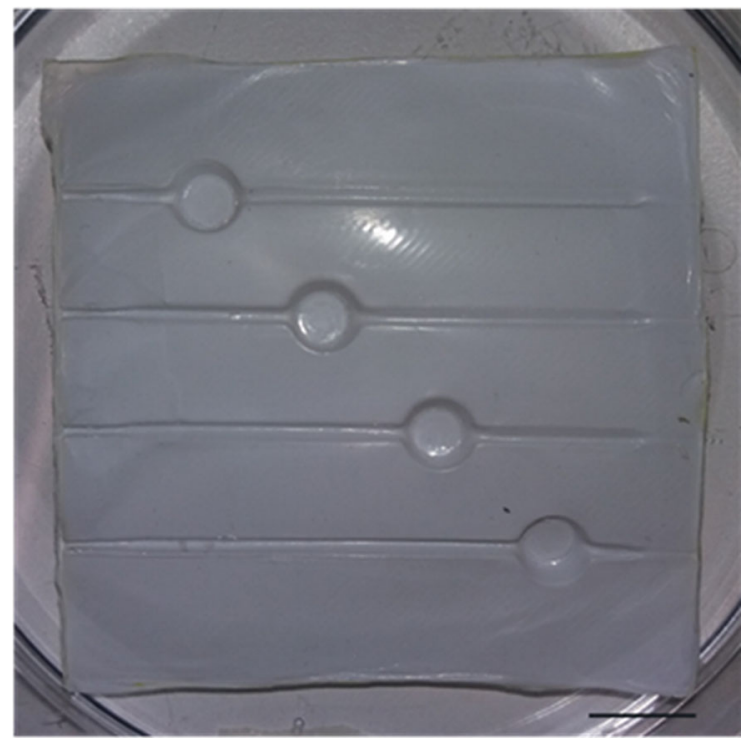

Fig. 8 BC platform obtained after embossing of BC between the two complementary molds. (Scale bar $=1 \mathrm{~cm}$ )

resistance and biocompatibility, can show a new array of applications.

\section{Conclusions}

In this work, a combination of two surface modification methods was used to allow the production of highly hydrophobic BC membranes. The use of oxygen plasma followed by silanization using TCMS allowed to obtain a higher roughness and lower surface energy, respectively. This resulted in a hydrophobic BC membrane that can withstand wet conditions for up to a month and has no short-term effects on cell viability, leading to the possibility of using this material as a platform for cell culture and microfluidic devices, replacing the use of common plastics.

Acknowledgments This work was supported by the Fundação para a Ciência e a Tecnologia (FCT), under the project "SkinChip: Disruptive cellulose-based microfluidic device for 3D skin modelling" (PTDC/BBB-BIO/1889/2014, SFRH/BPD/ 121526/2016), co-financed by the Lisboa 2020, COMPETE 2020, Portugal 2020 and the BioTecNorte operation (NORTE01-0145-FEDER-000004) with funding by the European Regional Development Fund under the scope of Norte2020Programa Operacional Regional do Norte.

\section{Compliance with ethical standard}

Conflict of interest No potential conflict of interest was reported by the authors

\section{References}

Bhushan B, Jung YC (2011) Effects of surface structure on wetting of patterned superhydrophobic surfaces. In: Drummond CH\& III (Eds.), 68th Conference on glass problems, vol. 29. Wiley

Bhushan B, Nosonovsky M (2010) The rose petal effect and the modes of superhydrophobicity. Philos Trans A Math Phys Eng Sci 368(1929):4713-4728. https://doi.org/10.1098/ rsta.2010.0203

Cacicedo ML, Castro MC, Servetas I, Bosnea L, Boura K, Tsafrakidou P, Castro GR et al (2016) Progress in bacterial cellulose matrices for biotechnological applications. Bioresour Technol 213:172-180. https://doi.org/10.1016/j. biortech.2016.02.071

Calvimontes A, Mauersberger P, Nitschke M, Dutschk V, Simon F (2011) Effects of oxygen plasma on cellulose surface. Cellulose 18(3):802-809. https://doi.org/10.1007/ s10570-011-9511-5

Cassie ABD, Baxter S (1944) Wettability of porous surfaces. Trans Faraday Soc 40:546-551. https://doi.org/10.1039/ TF9444000546

Costa MN, Veigas B, Jacob JM, Santos DS, Gomes J, Baptista PV, Fortunato E et al (2014) A low cost, safe, disposable, rapid and self-sustainable paper-based platform for diagnostic testing: lab-on-paper. Nanotechnology 25(9):094006. https://doi.org/10.1088/0957-4484/25/9/ 094006

Costa AFS, Almeida FCG, Vinhas GM, Sarubbo LA (2017) Production of bacterial cellulose by gluconacetobacter hansenii using corn steep liquor as nutrient sources. Front Microbiol 8:2027. https://doi.org/10.3389/fmicb.2017. 02027

Creighton JR, Ho P (2010) Introduction to Chemical Vapour Depositionl SpringerLink. In: Xu Y, Yan X-T (eds) engineeing materials and processes. Springer, London, pp 1-28

Fadeev AY, McCarthy TJ (2000) Self-Assembly Is Not the Only Reaction Possible between Alkyltrichlorosilanes and Surfaces: monomolecular and Oligomeric Covalently Attached Layers of Dichloro-and Trichloroalkylsilanes on Silicon. Langmuir 16(18):7268-7274. https://doi.org/ $10.1021 / 1 \mathrm{a} 000471 \mathrm{z}$

Faria G, Cardoso CR, Larson RE, Silva JS, Rossi MA (2009) Chlorhexidine-induced apoptosis or necrosis in L929 fibroblasts: a role for endoplasmic reticulum stress. Toxicol Appl Pharmacol 234(2):256-265. https://doi.org/10.1016/ j.taap.2008.10.012

Fink H, Faxalv L, Molnar GF, Drotz K, Risberg B, Lindahl TL, Sellborn A (2010) Real-time measurements of coagulation on bacterial cellulose and conventional vascular graft materials. Acta Biomater 6(3):1125-1130. https://doi.org/ 10.1016/j.actbio.2009.09.019

Glavan AC, Martinez RV, Subramaniam AB, Yoon HJ, Nunes RMD, Lange H, Whitesides GM et al (2013) Omniphobic 
"RF Paper" Produced by Silanization of Paper with Fluoroalkyltrichlorosilanes. Adv Func Mater 24:60-70

Helenius G, Backdahl H, Bodin A, Nannmark U, Gatenholm P, Risberg B (2006) In vivo biocompatibility of bacterial cellulose. J Biomed Mater Res A 76(2):431-438. https:// doi.org/10.1002/jbm.a.30570

Hong X, Gao X, Jiang L (2007) Application of Superhydrophobic Surface with High Adhesive Force in No Lost Transport of Superparamagnetic Microdroplet. J Am Chem Soc 129(6):1478-1479. https://doi.org/10.1021/ja065537c

Huang Y, Zhu C, Yang J, Nie Y, Chen C, Sun D (2014) Recent advances in bacterial cellulose. Cellulose 21(1):1-30

ISO 10993-5. (2009). Biological evaluation of medical devices. In: Part 5: Tests for in vitro cytotoxicity, 3rd ed. pp 34)

Jedrzejczak-Krzepkowska M, Kubiak K, Ludwicka K, Bielecki S (2016) Chapter 2-bacterial nanocellulose synthesis, recent findings. In: Gama M, Dourado F, Bielecki S (eds) Bacterial nanocel-lulose: from biotechnology to bioeconomy. Elsevier, New York, pp 19-46

Katara G, Hemvani N, Chitnis S, Chitnis V, Chitnis DS (2008) Surface disinfection by exposure to germicidal UV light. Indian J Med Microbiol 26(3):241-242

Klemm D, Heublein B, Fink HP, Bohn A (2005) Cellulose: fascinating biopolymer and sustainable raw material. Angew Chem Int Ed Engl 44(22):3358-3393. https://doi. org/10.1002/anie.200460587

Kumar A, Negi YS, Choudhary V, Bhardwaj NK (2014) Characterization of cellulose nanocrystals produced by acid-hydrolysis from sugarcane bagasse as agro-waste. J Mater Phys Chem 2(1):1-8

Leitao AF, Faria MA, Faustino AM, Moreira R, Mela P, Loureiro L, Gama M et al (2016) A novel small-caliber bacterial cellulose vascular prosthesis: production, characterization, and preliminary in vivo testing. Macromol Biosci 16(1):139-150. https://doi.org/10.1002/mabi.201500251

Mohd NH, Ismail NFH, Zahari JI, Wan Fathilah WFb, Kargarzadeh H, Ramli S, Othaman R et al (2016) Effect of aminosilane modification on nanocrystalline cellulose properties. J Nanomater 2016 2016:8. https://doi.org/10. $1155 / 2016 / 4804271$

Mosmann T (1983) Rapid colorimetric assay for cellular growth and survival: application to proliferation and cytotoxicity assays. J Immunol Methods 65(1-2):55-63. https://doi.org/ 10.1016/0022-1759(83)90303-4

North EJ, Halden RU (2013) Plastics and environmental health: the road ahead. Rev Environ Health 28(1):1-8

Pan C, Kumar C, Bohl S, Klingmueller U, Mann M (2009) Comparative proteomic phenotyping of cell lines and primary cells to assess preservation of cell type-specific functions. Mol Cell Proteomics 8(3):443-450. https://doi. org/10.1074/mcp.M800258-MCP200

Petersen N, Gatenholm P (2011) Bacterial cellulose-based materials and medical devices: current state and perspectives. Appl Microbiol Biotechnol 91(5):1277-1286. https://doi.org/10.1007/s00253-011-3432-y

Picheth GF, Pirich CL, Sierakowski MR, Woehl MA, Sakakibara CN, de Souza CF, de Freitas RA et al (2017) Bacterial cellulose in biomedical applications: a review. Int J Biol
Macromol 104:97-106. https://doi.org/10.1016/j.ijbiomac. 2017.05.171

Rachini A, Le Troedec M, Peyratout C, Smith A (2012) Chemical modification of hemp fibers by silane coupling agents. J Appl Polym Sci 123(1):601-610. https://doi.org/ 10.1002/app.34530

Riss TL, Moravec RA, Niles AL, Duellman S, Benink HA, Worzella TJ, Minor L (2016) Cell viability assays. In: Assay guidance manual: Eli Lilly \& Company and the National Center for Advancing Translational Sciences

Seo K, Kim M, Kim DH (2015) Re-derivation of Young's equation, Wenzel equation, and Cassie-Baxter equation based on energy minimization. In: Aliofkhazraei M (ed) Surface energy. IntechOpen, London

Shao W, Wu J, Liu H, Ye S, Jiang L, Liu X (2017) Novel bioactive surface functionalization of bacterial cellulose membrane. Carbohyd Polym 178:270-276

Silva MA, Leite YKC, de Carvalho CES, Feitosa MLT, Alves MMM, Carvalho FAA, de Carvalho MAM et al (2018) Behavior and biocompatibility of rabbit bone marrow mesenchymal stem cells with bacterial cellulose membrane. PeerJ 6:e4656. https://doi.org/10.7717/peerj.4656

Song J, Rojas OJ (2013) Approaching super-hydrophobicity from cellulosic materials: a review. Nord Pulp Pap Res J 28(2):216-238

Svensson A, Nicklasson E, Harrah T, Panilaitis B, Kaplan DL, Brittberg M, Gatenholm P (2005) Bacterial cellulose as a potential scaffold for tissue engineering of cartilage. Biomaterials 26(4):419-431. https://doi.org/10.1016/j. biomaterials.2004.02.049

Thuo MM, Martinez RV, Lan W-J, Liu X, Barber J, Atkinson MBJ, Whitesides GM et al (2014) Fabrication of low-cost paper-based microfluidic devices by embossing or cut-andstack methods. Chem Mater 26(14):4230-4237

Wang Y-W, Wu Q, Chen G-Q (2003) Reduced mouse fibroblast cell growth by increased hydrophilicity of microbial polyhydroxyalkanoates via hyaluronan coating. Biomaterials 24(25):4621-4629. https://doi.org/10.1016/S01429612(03)00356-9

Wenzel RN (1936) Resistance of solid surfaces to wetting by water. Ind Eng Chem 28(8):988-994. https://doi.org/10. 1021/ie50320a024

Zhang H, Li Y, Xu Y, Lu Z, Chen L, Huang L, Fan M (2016) Versatile fabrication of a superhydrophobic and ultralight cellulose-based aerogel for oil spillage clean-up. Phys Chem Chem Phys 18(40):28297-28306. https://doi.org/10. 1039/C6CP04932J

Zimmermann KA, LeBlanc JM, Sheets KT, Fox RW, Gatenholm P (2011) Biomimetic design of a bacterial cellulose/ hydroxyapatite nanocomposite for bone healing applications. Mater Sci Eng C 31(1):43-49. https://doi.org/10. 1016/j.msec.2009.10.007

Publisher's Note Springer Nature remains neutral with regard to jurisdictional claims in published maps and institutional affiliations. 